\section{RICYDE. Revista Internacional de Ciencias del Deporte doi: $10.5232 /$ ricyde \\ Rev. Int. cienc. deporte}

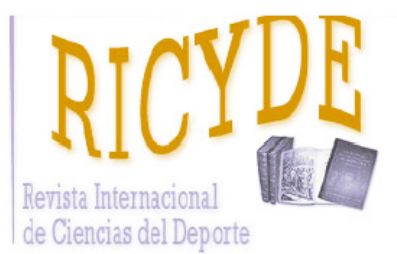

RICYDE. Revista Internacional de Ciencias del Deporte Volumen XIV - Año XIV

Páginas: 321-333 - ISSN: 1885-3137

Número 54 - octubre 2018

\title{
Juego y práctica deliberada en la formación deportiva de jugadores de élite del voleibol \\ Play and practice in sport development of volleyball elite athletes
}

\author{
Carine Collet ${ }^{1}$, Juarez Vieira do Nascimento ${ }^{1}$, Alexandra Folle ${ }^{2}$, \\ Felipe Goedert Mendes ${ }^{1}$ y Sergio José Ibáñez ${ }^{3}$
}

1. Universidade Federal de Santa Catarina. Brazil

2. Universidade do Estado de Santa Catarina. Brazil

3. Universidad de Extremadura. España

\section{Resumen}

El objetivo de este estudio fue analizar el tiempo de práctica semanal en la participación en actividades de juego deliberado y práctica deliberada durante las etapas de formación deportiva de jugadores de élite del voleibol brasileño. Participaron del estudio 121 jugadores de las selecciones brasileñas de voleibol de las categorías de formación y adultas, así como jugadores que ya finalizaron su carrera deportiva. Los datos relacionados a las horas semanales de participación en las actividades en cada etapa de formación deportiva se obtuvieron a través de un cuestionario. El análisis de datos se realizó a partir de la MLG-ANOVA de dos factores. Los resultados indicaron equilibrio en la duración semanal de la participación en actividades de juego y práctica deliberada en los años de diversificación, mientras que se observó diferencia significativa $(p<0,05)$ en la duración de cada tipo de actividad entre los años de especialización e inversión. Se observó un aumento gradual del desarrollo con la práctica deliberada y, consecuentemente, la disminución de la participación con el juego deliberado en la evolución de las etapas. Se concluye que estos jugadores se involucraron en actividades de práctica deliberada desde el inicio de la formación deportiva, un hecho que puede haber influido en su desempeño como jugador de voleibol, a partir de vivencias con el deporte sistematizado.

Palabras clave: Desarrollo deportivo; participación en actividades; etapas de la participación deportiva.

\section{Abstract}

The aim of this study was to analyze the weekly practice time in the participation in deliberate play and deliberate practice activities during the stages of sports development of elite players of Brazilian volleyball. Participating in the study 121 players from the Brazilian volleyball national teams of the training and adult categories, as well as players who had already finished their sporting career. The data related to the weekly hours of participation in the activities in each stage of sports training were obtained through a questionnaire. The data analysis was performed from the GLM-ANOVA two-factor. The results indicated a balance in the weekly duration of participation in deliberate play and deliberate practice activities in the sampling years, while there was a significant difference $(p<0.05)$ in the duration of each type of activity between the specialization and investment years. A gradual increase of the development was observed with the deliberate practice and, consequently, the decrease of the participation in deliberate play in the evolution of the stages. It is concluded that these players were involved in activities of deliberate practice since the beginning of sports development, a fact that may have influenced their performance as a volleyball player, from experiences with systematized sport.

Key Words: Sports development; engagement in activities; stages of sport participation.

Correspondencia/correspondence: Carine Collet Universidade Federal de Santa Catarina. Brazil

Email: carine.collet5@gmail.com 
Collet, C.; Nascimento, J. V.; Folle, A.; Mendes, F. G., y Ibañez, S. J. (2018). Juego y práctica deliberada en la formación deportiva de jugadores de élite del voleibol. RICYDE. Revista internacional de ciencias del deporte. 54(14), 321-333. https://doi.org/10.5232/ricyde2018.05403

\section{Introducción}

A lcanzar el rendimiento de élite en el deporte se constituye en un camino largo y arduo, que requiere dedicación, superación e inserción en programas orientados hacia la formación deportiva. Implicarse en diferentes tipos de actividades físicas y deportivas durante la infancia podrá influenciar positivamente en la práctica deportiva sistematizada en la adolescencia y en la edad adulta, teniendo en cuenta que la iniciación deportiva normalmente ocurre en una fase de transición de la infancia a la edad adulta (Côté, Lidor y Hackfort, 2009, Mesquita, 1998, Milistetd, Mesquita, Nascimento y Souza Sobrinho, 2010). Los momentos con actividades lúdicas y autorreguladas (juego deliberado), combinadas con actividades orientadas al rendimiento específico (práctica deliberada), son la base para la formación deportiva.

El juego deliberado consiste en un conjunto de actividades físicas y experiencias de movimientos espontáneos y autónomos, de diferentes juegos y bromas que se practican en las calles, parques y en entornos no formales (Côté, 1999; Côté, Baker y Abernethy, 2003; Côté y Fraser-Thomas, 2007), correspondientes a deportes adaptados y teniendo como principales características la diversión, motivación y autorregulación (Santos, 2011). Por otro lado, las actividades de práctica deliberada corresponden a la ejercitación individualizada de actividades específicas de entrenamiento, planeadas para mejorar aspectos del desempeño deportivo del individuo, a través de la repetición y del refinamiento sucesivo, apoyados en constante supervisión y emisión de feedbacks por parte del conductor del proceso (Ericsson, 2003, Ericsson, Krampe y Tesch-Romer, 1993). Tales actividades proporcionan la adquisición y el refinamiento de movimientos específicos de un deporte $\mathrm{y}$, consecuentemente, la búsqueda por altos niveles de desempeño deportivo y rendimiento de élite.

En este contexto, la literatura confirma la necesidad de unión entre juego y práctica deliberada durante la formación deportiva (Baker, Côté y Abernethy, 2003a; Côté, 1999; Côté y col., 2003; Côté, Baker y Abernethy, 2007; Ericsson, 2006), en la que la motivación y las vivencias de uno ayudan en el desarrollo y evolución del otro. En muchos casos, después de la fase inicial con experiencias motoras generalizadas, los jugadores pasan a comprometerse con contextos específicos, a través de la práctica deliberada para perfeccionar sus habilidades deportivas.

El predominio del tipo de actividad realizada en cada etapa de la formación deportiva se describe a partir de diferentes modelos e indicaciones, con el propósito de hacer del deporte una herramienta que ayuda en el desarrollo humano. El Developmental Model of Sport Participation, o Modelo de Desarrollo de la Participación Deportiva (MDPD) (Côté, 1999, Côté y col., 2003, Côté y Fraser-Thomas, 2007), elaborado a partir de los conceptos teóricos de juego deliberado, práctica deliberada y etapas de la formación deportiva, se configura como una herramienta que busca el desarrollo armonioso de los niños durante su trayectoria deportiva. Las indicaciones sobre las etapas de formación deportiva poseen características específicas, tanto en lo que se refiere a la diversificación y la especialización deportiva, como en la participación en actividad de práctica y juego deliberado (Côté y Abernethy, 2012; Côté y col., 2009).

El MDPD ha sido uno de los principales modelos que aclara la importancia de patrones de entrenamiento y de las influencias sociales durante el desarrollo deportivo (Côté, 1999; Côté y col., 2009). En general, el modelo destaca la importancia de la vivencia en las prácticas de diferentes deportes y la gran cantidad de actividades de juego deliberado en los años de diversificación, el inicio de la inserción en actividades de práctica deliberada mezclando con actividades de juego deliberado en los años de especialización y, a partir de los años de 
Collet, C.; Nascimento, J. V.; Folle, A.; Mendes, F. G., y Ibañez, S. J. (2018). Juego y práctica deliberada en la formación deportiva de jugadores de élite del voleibol. RICYDE. Revista internacional de ciencias del deporte. 54(14), 321-333. https://doi.org/10.5232/ricyde2018.05403

inversión, la participación más intensa en actividad de práctica deliberada, invirtiendo esfuerzos en el perfeccionamiento de un deporte específico (Côté y Abernethy, 2012; Côté y col., 2003; Côté y col., 2009).

El modelo surgió a partir de investigaciones con jugadores de diferentes deportes, presentando posibilidades de trayectorias relacionadas con la participación recreacional, al rendimiento de élite por medio de la diversificación deportiva, y al rendimiento de élite por medio de la especialización precoz (Côté y Fraser-Thomas, 2007). Las dos primeras trayectorias presentan posibilidades más grandes de mantenimiento de la práctica deportiva a lo largo de la vida, teniendo en cuenta que pueden traer beneficios en términos psicosociales y de salud. Contrariamente, la tercera trayectoria, enfocada en la especialización precoz, puede llevar al deporte de élite, pero también resultar negativa para la salud física y la diversión, además de abandono y alejamiento prematuro del deporte (Côté, 1999; Côté y col., 2007; Côté y Fraser-Thomas, 2007; Côté y Hay, 2002; Côté y Vierimaa, 2014).

Diferentes estudios son realizados con el propósito de comprobar la participación en actividades de juego y práctica deliberada en las etapas de la formación deportiva de jugadores de selecciones nacionales (Baker y col., 2003a, Gullich, 2016), y de diferentes deportes colectivos (Forsman, Blomqvist, Davids, Konttinen e Liukkonen, 2016; Memmert, Baker e Bertsch, 2010; Vink, Raudsepp e Kais, 2015), Además, algunos estudios fueron realizados con jugadores de diferentes deportes, como el fútbol (Berry, Abernethy y Côté, 2008, Hendry, Crocker y Hodges, 2014, Hornig, Aust y Gullich, 2016, Ward, Hodges, Starkes y Williams, 2007), el hockey sobre hielo (Soberlak y Côté, 2003), atletismo (CasadoAlda y Ruiz-Pérez, 2017), y voleibol (Coutinho, Mesquita, Davids, Fonseca y Côté, 2016).

Las etapas de formación deportiva se clasifican, según Côté (1999), en los años de diversificación (6 a 12 años), especialización (13 a 15 años) e inversión (16 a 18 años). El camino hasta alcanzar la élite en un deporte sugiere la elevada participación en actividades de juego deliberado en los años de diversificación, pasando a la participación gradual en actividades de práctica deliberada en los años de especialización e inversión. En este caso, la especialización se inicia en torno a los 13 años, después de la participación en distintas actividades de práctica y juego deliberado, mientras que la inversión aumenta el compromiso con solamente una actividad de práctica deliberada (Côté y Abernethy, 2012).

Teniendo en cuenta tales estudios y modelos internacionalmente conocidos, se despierta el interés de como esto proceso se ha desarrollado en el contexto brasileño. Uno de los deportes más practicados en Brasil es el voleibol, que se ha destacado a partir de conquistas en campeonatos internacionales y por el alto nivel de excelencia de sus jugadores, despertando el interés sobre su proceso de formación deportiva. En vista de la escasa información publicada sobre el desarrollo de jugadores del voleibol en Brasil, el presente estudio tuvo como objetivo analizar la formación deportiva de jugadores de élite del voleibol brasileño, analizando el tiempo de práctica semanal en la participación en actividades de juego deliberado y práctica deliberada durante las etapas de formación deportiva.

\section{Método}

El presente estudio corresponde a una investigación empírica con estrategia asociativa, así como un estudio comparativo retrospectivo, pues la ocurrencia de las variables se dio antes del inicio de la investigación, puesto que sólo requiere registrar y analizar los datos (Ato, López y Benavente, 2013). 
Collet, C.; Nascimento, J. V.; Folle, A.; Mendes, F. G., y Ibañez, S. J. (2018). Juego y práctica deliberada en la formación deportiva de jugadores de élite del voleibol. RICYDE. Revista internacional de ciencias del deporte. 54(14), 321-333. https://doi.org/10.5232/ricyde2018.05403

\section{Participantes}

Participaron del estudio 121 jugadores de las selecciones brasileñas femeninas de las categorías sub18 $(n=18)$, sub21 $(n=22)$ y sub23 $(n=19)$, y masculinas de las categorías sub19 $(n=19)$, sub21 $(n=19)$, sub21 $(n=18)$, sub21 $(n=19)$, sub21 $(n=19)$, como de las selecciones sénior $(n=5)$ y jugadores masculinos que ya finalizaron su carrera deportiva $(n=19)$.

\section{Instrumento}

El instrumento utilizado en la recogida de datos fue un cuestionario que permite tener información sobre la formación deportiva de jugadores de voleibol. El cuestionario está dividido en dos partes: la primera relacionada al perfil sociodemográfico y la segunda subdividida las etapas de formación deportiva (Côté, 1999). En cada etapa, las cuestiones abordan los elementos dinámicos de la formación deportiva (Côté, Turnnidge y Vierimaa, 2016), relacionados con las características de las actividades realizadas, las relaciones de incentivo y los contextos de práctica. El proceso de validación de contenido, estabilidad y consistencia interna del instrumento reveló altos índices en todos los aspectos. La validación de contenido, realizada por la evaluación de expertos, obtuvo Índice de Validez de Contenido $V$ Aiken (Aiken, 1985) de ,91. La estabilidad fue significativa en 77 de los 78 ítems y la consistencia interna presentó coeficiente $\alpha>, 70$ en 77 de los 78 ítems del cuestionario.

En el presente estudio, las variables analizadas fueron: etapas de la formación deportiva (diversificación, especialización, inversión) y duración (en horas por semana) de las actividades juego deliberado y práctica deliberada. Para eso, fue utilizada las siguientes preguntas para cada etapa de la formación (Tabla 1).

Tabla 1. Preguntas utilizadas en el estudio para obtener las informaciones sobre las horas de juego y practica deliberada.

1. Indique las actividades que usted practicaba hasta los 12 años y, para cada una de ellas, indique la característica de la actividad, cuántas horas a la semana y marque el número que corresponde a su motivación personal.

\begin{tabular}{|c|c|c|c|c|c|c|c|}
\hline \multirow{2}{*}{$\begin{array}{c}\text { Actividades físicas o } \\
\text { deportivas practicadas } \\
\text { (incluso juegos de calle) }\end{array}$} & \multirow{2}{*}{$\begin{array}{c}\text { Característica (ocio o } \\
\text { entrenamiento) }\end{array}$} & \multirow{2}{*}{$\begin{array}{c}\text { Cuántas horas a la } \\
\text { semana } \\
\text { (aproximadamente) }\end{array}$} & \multicolumn{5}{|c|}{$\begin{array}{l}\text { Motivación personal para la } \\
\text { práctica }\end{array}$} \\
\hline & & & $\begin{array}{l}\text { Muy } \\
\text { baja }\end{array}$ & Baja & Moderado & Alta & $\begin{array}{c}\mathrm{Mu} \\
\mathrm{y} \\
\text { alta }\end{array}$ \\
\hline 1- & $\begin{array}{c}\text { ( ) ocio } \\
\text { ( ) entrenamiento }\end{array}$ & & 1 & 2 & 3 & 4 & 5 \\
\hline $2-$ & $\begin{array}{c}\text { ( ) ocio } \\
\text { ( ) entrenamiento }\end{array}$ & & 1 & 2 & 3 & 4 & 5 \\
\hline $3-$ & $\begin{array}{c}\text { ( ) ocio } \\
\text { ( ) entrenamiento }\end{array}$ & & 1 & 2 & 3 & 4 & 5 \\
\hline 4- & $\begin{array}{c}\text { ( ) ocio } \\
\text { ( ) entrenamiento }\end{array}$ & & 1 & 2 & 3 & 4 & 5 \\
\hline 5- & $\begin{array}{c}\text { ( ) ocio } \\
\text { ( ) entrenamiento }\end{array}$ & & 1 & 2 & 3 & 4 & 5 \\
\hline
\end{tabular}

\section{Procedimientos}

La realización del estudio fue aprobada por el Comitê de Ética em Pesquisa com Seres Humanos de una universidad pública de Brasil (parecer 1.450.060/2016). Los jugadores con más de 18 años firmaron el Termo de Consentimiento Libre y Esclarecido y los jugadores 
Collet, C.; Nascimento, J. V.; Folle, A.; Mendes, F. G., y Ibañez, S. J. (2018). Juego y práctica deliberada en la formación deportiva de jugadores de élite del voleibol. RICYDE. Revista internacional de ciencias del deporte. 54(14), 321-333. https://doi.org/10.5232/ricyde2018.05403

menores de 18 años firmaron el Termo de Asentimiento acompañado del Termo de Consentimiento firmado por los padres o responsables legales.

La aplicación de cuestionario en los jugadores de las categorías de base se realizó presencialmente, en el Centro de Desarrollo de Voleibol de la Conferedação Brasileira de Voleibol durante los meses de julio y agosto de 2016. Se obtuvieron también respuestas on line, a partir de la herramienta Google Docs, por la imposibilidad de encuentros presenciales con los jugadores en edad sénior y los que finalizaron su carrera deportiva.

\section{Fiabilidad de los datos}

La fiabilidad de la información emitida por los jugadores se comprobó en el 11,6\% de la muestra $(n=4)$, a partir de la replicación del cuestionario seis meses después de la primera recogida de datos. El análisis fue realizado por el Índice de Correlación Intraclase entre los datos del test y del retest (Field, 2009). La cantidad de horas de práctica deliberada recogidas en la segunda aplicación presentó media de 3,71 \pm 8 horas menos que en la primera toma de datos $(r=, 82, I C 95 \%=, 59$ a, 93$)$, y la cantidad de juego deliberado, presentó una disminución media de 3,29 $\pm 5,53$ horas entre las aplicaciones $(r=, 89, I C 95 \%=, 74$ a ,96). Sin embargo, los altos valores de correlación entre las informaciones registradas en los dos momentos de aplicación del cuestionario confirman la fiabilidad de los datos.

\section{Análisis estadístico}

Inicialmente se realizó un análisis descriptivo para la identificación y exclusión de los casos con valores extremos, que son valores numéricamente distantes del resto de los datos, identificados a partir del programa estadístico SPSS versión 23 (SPSS Inc., Chicago IL, USA). Se consideraron un total de 102 casos filtrados para los análisis estadísticos. En secuencia, los valores de media y desviación típica de las horas de participación en actividades de juego y práctica deliberada se determinaron en cada etapa de la formación deportiva, así como un análisis exploratorio se realizó para verificar la homogeneidad de variancias a partir del Test de Levene (Field, 2009). Las variables que no presentaron homogeneidad de varianzas fueron transformadas a partir de la prueba de Box Cox (Box y Cox, 1964), con un valor de transformación de ,275. Finalmente, el análisis comparativo de los datos fue realizado a partir del análisis de varianzas de dos factores con medidas repetidas (Modelo Lineal General - ANOVA) para verificar la variación a lo largo del tiempo, y del Test de Post-Hoc con ajuste de intervalo de Bonferroni, adoptando el nivel de significación de 5\% $(p<, 05)$.

Los niveles de significación se analizaron con la cuantificación de la potencia $(\phi)$ y del tamaño del efecto (eta-square values - $\eta^{2}$ ), el que permite comprender adecuadamente los resultados de dichos análisis y constituye una prueba más relevante para la validez del estudio (Cárdenas y Arancibia, 2014). Estimar el tamaño del efecto corresponde a la magnitud de las diferencias encontradas en el estudio, la potencia estadística corresponde al grado de validez que poseen los resultados de la investigación (Cohen, 1992; Field, 2009, Grissom y Kim, 2012). Todos los análisis se realizaron con la ayuda del programa estadístico SPSS versión 23 (SPSS Inc., Chicago IL, USA). La significación estadística se estableció en $p<, 05$.

\section{Resultados}

Los resultados revelaron aumento en la duración en horas de actividades de práctica deliberada durante las etapas de la formación deportiva, al mismo tiempo que hubo una disminución gradual de la duración semanal en la participación con actividades de juego deliberado. La Tabla 2 demuestra la variación de la duración de cada tipo de actividad, relacionada con las etapas de la formación deportiva. 
Collet, C.; Nascimento, J. V.; Folle, A.; Mendes, F. G., y Ibañez, S. J. (2018). Juego y práctica deliberada en la formación deportiva de jugadores de élite del voleibol. RICYDE. Revista internacional de ciencias del deporte. 54(14), 321-333. https://doi.org/10.5232/ricyde2018.05403

Tabla 2. Resultados descriptivos y diferencias en la duración de cada tipo de actividad en cada etapa de la formación deportiva.

\begin{tabular}{lccccccccccccccc}
\hline \multicolumn{11}{c}{$\begin{array}{c}\text { Años de } \\
\text { diversificación }\end{array}$} & \multicolumn{1}{c}{$\begin{array}{c}\text { Años de } \\
\text { especialización }\end{array}$} & \multicolumn{1}{c}{ Años de inversión } \\
\hline & $M$ & $D T$ & $E T$ & $M$ & $D T$ & $E T$ & $M$ & $D T$ & $E T$ & $F$ & $g l$ & $g l$ & $p$ & $n^{2}$ & $\phi$ \\
\hline $\begin{array}{l}\text { Horas de práctica } \\
\text { deliberada }\end{array}$ & 6,31 & 5,50 & 0,54 & 10,02 & 5,90 & 0,58 & 14,34 & 6,78 & 0,67 & 87.724 & 1 & 90 & $<, 001$ &, 465 & 1 \\
$\begin{array}{l}\text { Horas de juego } \\
\text { deliberado }\end{array}$ & 6,79 & 5,61 & 0,56 & 4,12 & 5,27 & 0,52 & 1,62 & 2,96 & 0,29 & 66.688 & 1 & 90 & $<, 001$ &, 398 & 1 \\
\hline
\end{tabular}

Las diferencias entre las etapas de la formación deportiva, tanto en el aumento de la cantidad de horas de práctica deliberada, como en la disminución de las horas de juego deliberado, revelaron valores de potencia óptimos para ambas variables $(\phi>, 80)$. En el caso del tamaño del efecto, según Cárdenas y Arancibia (2014), las horas de práctica deliberada presentan un tamaño de efecto grande $\left(\eta^{2}>, 40\right)$, mientras que las horas de juego deliberado presentaron un tamaño de efecto medio $\left(\eta^{2}>, 25\right)$.

La Tabla 3 presenta las diferencias específicas entre las etapas, obtenidas por medio de la prueba de Post Hoc, considerando las actividades de juego y práctica deliberada, presentando diferencias significativas entre todos los períodos analizados.

Tabla 3. Comparación entre las etapas de la formación deportiva, considerando las horas de juego y práctica deliberada.

\begin{tabular}{llcc}
\hline & & $\begin{array}{c}\text { Horas de juego } \\
\text { deliberado }\end{array}$ & $\begin{array}{c}\text { Horas de práctica } \\
\text { deliberada }\end{array}$ \\
\hline Años de diversificación & Años de especialización & $p$ & $p$ \\
\hline Años de especialización & Años de inversión & $<, 001$ & $<, 001$ \\
& Años de diversificación & $<, 001$ & $<, 001$ \\
\hline Años de inversión & Años de inversión & $<, 001$ & $<, 001$ \\
& Años de diversificación & $<, 001$ & $<, 001$ \\
\hline
\end{tabular}

La Figura 1 representa gráficamente la característica de las actividades de juego y práctica deliberada a lo largo de las etapas de la formación deportiva, poniendo de manifiesto las diferencias estadísticamente significativas entre las horas de juego y práctica deliberada en los años de especialización, lo que no ocurrió en los años de diversificación. 
Collet, C.; Nascimento, J. V.; Folle, A.; Mendes, F. G., y Ibañez, S. J. (2018). Juego y práctica deliberada en la formación deportiva de jugadores de élite del voleibol. RICYDE. Revista internacional de ciencias del deporte. 54(14), 321-333. https://doi.org/10.5232/ricyde2018.05403

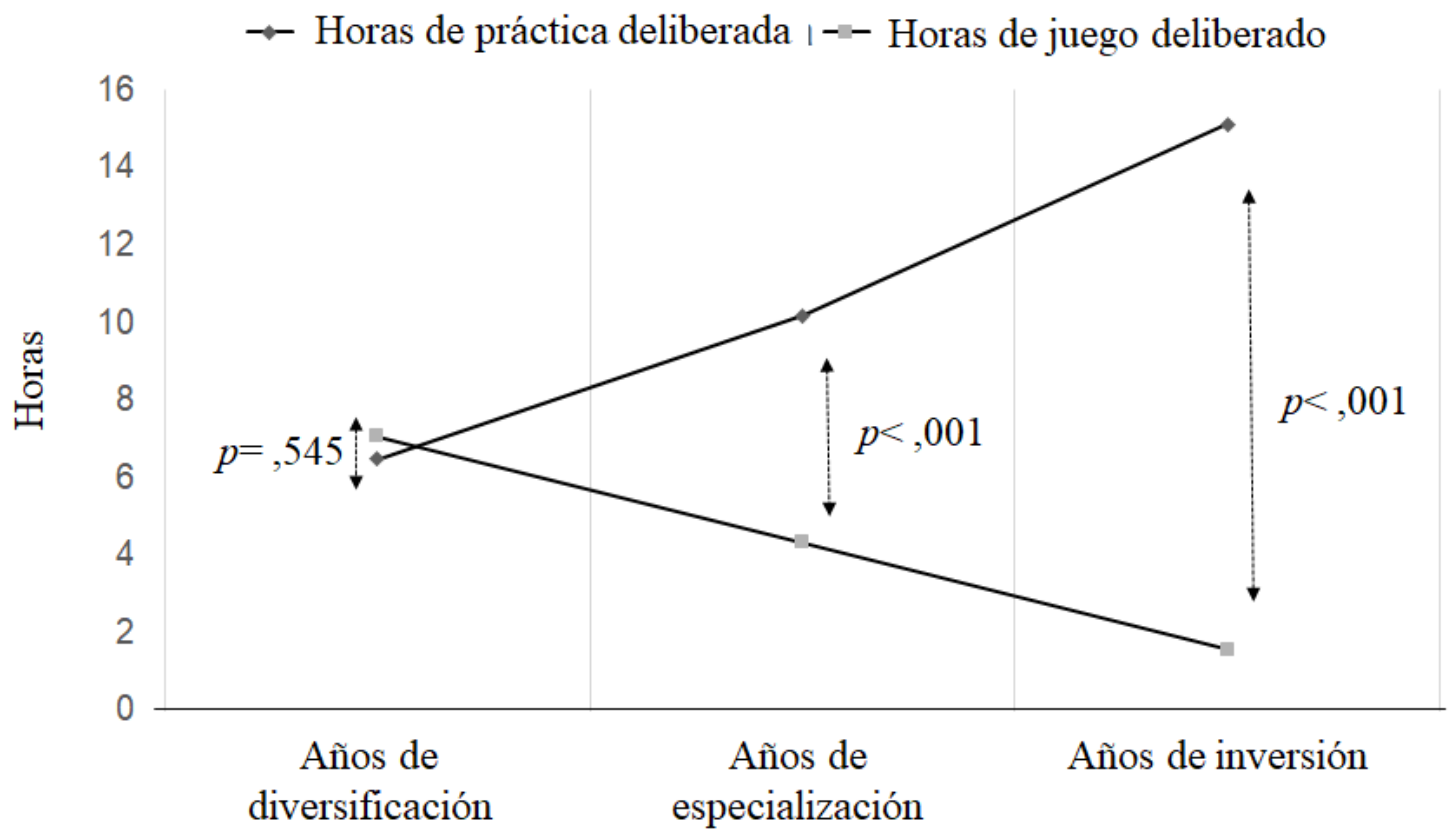

Figura 1: Variación en la cuenta de las actividades de juego y práctica deliberada a lo largo de las etapas de formación deportiva.

\section{Discusión}

El objetivo del estudio fue analizar el tiempo de práctica semanal en la participación en actividades de juego y práctica deliberada durante las etapas de formación deportiva de jugadores de élite del voleibol brasileño. Se constató el equilibrio entre las horas de actividades de juego y práctica deliberada en los años de diversificación y posteriormente un aumento significativo y gradual en las horas de práctica deliberada $\mathrm{y}$, consecuentemente, disminución en las horas de juego deliberado a lo largo de la formación deportiva. En general, la formación de los jugadores de élite del voleibol brasileño ha demostrado gran variabilidad de características, algunas de ellas se aproximan y otras se diferencian de modelos internacionalmente conocidos.

Las horas de participación en el juego deliberado pueden haber influido positivamente en la formación deportiva de los jugadores investigados, teniendo en cuenta que tales actividades favorecen el entusiasmo con el deporte, pudiendo llevar a buenas oportunidades para la posterior adquisición de habilidades específicas (Baker y Young, 2014; Côté y Erickson, 2015; Memmert y col., 2010). Estas actividades mejoran la percepción, la creatividad, la toma de decisiones y la motivación, siendo discriminantes entre grupos de expertos y no expertos (Memmert y col., 2010). Además, participar de diferentes deportes en la fase inicial del desarrollo deportivo no perjudica la posibilidad de alcanzar el rendimiento en la élite, por el contrario, aumenta la participación y el compromiso (Diogo y Gonçalves, 2014).

La participación en la práctica deliberada se conforma con actividades estructuradas orientadas al desarrollo de la pericia deportiva se inició en los años de diversificación de los jugadores de voleibol brasileños. Estas actividades requieren esfuerzo y altos niveles de concentración, con características que diferencian la práctica deliberada de otras actividades como el desempeño de rutina o actividades como juegos y bromas dentro del dominio deportivo (Ericsson y col., 1993). En el caso de los deportistas, estas actividades se reconocen como agradables, a diferencia del concepto original definido a partir de músicos expertos, en que esas actividades no serían inherentemente motivantes (Baker y Young, 2014, Vink y col., 
Collet, C.; Nascimento, J. V.; Folle, A.; Mendes, F. G., y Ibañez, S. J. (2018). Juego y práctica deliberada en la formación deportiva de jugadores de élite del voleibol. RICYDE. Revista internacional de ciencias del deporte. 54(14), 321-333. https://doi.org/10.5232/ricyde2018.05403

2015). Los estudios han confirmado la relación positiva entre las horas de práctica deliberada y la de rendimiento deportivo, y destacan también la importancia de la unión entre el juego y la práctica deliberada en la formación de jugadores (Baker y col., 2003a; Berry y col., 2008; Memmert, 2010).

La cantidad de horas semanales de participación en actividades de juego y de práctica deliberada debe alternar en el transcurso de la evolución deportiva. En el caso del presente estudio, el aumento en las horas de práctica y la disminución de las horas de juego deliberado a lo largo de las etapas de formación deportiva están de acuerdo con las recomendaciones del MDPD y son similares a los estudios con jugadores de hockey sobre hielo (Soberlak y Côté, 2003) y de fútbol (Ford, Ward, Hodges y Williams, 2009). Por contra, los datos obtenidos de los jugadores brasileños, sobre de la cantidad de horas de juego y práctica dentro de cada etapa, fueron diferentes, en algunos aspectos, de las orientaciones del MDPD. Al analizar los datos de cada etapa en separado, se observó un equilibrio en las horas de juego y práctica deliberada en los años de diversificación, momento en que el modelo indica que haya más horas de juego en detrimento de la práctica deliberada. La Figura 2 representa gráficamente las diferencias encontradas a partir de una adaptación de las orientaciones del MDPD (izquierda) y de acuerdo con las informaciones obtenidas junto a los jugadores de élite del voleibol brasileño (derecha).

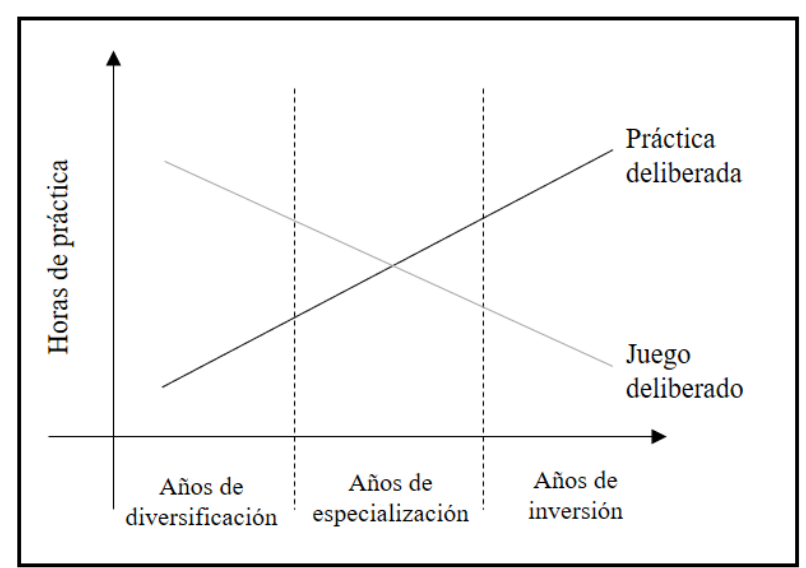

Adaptación en una representación gráfica de la formación deportiva a partir de las orientaciones del DMSP

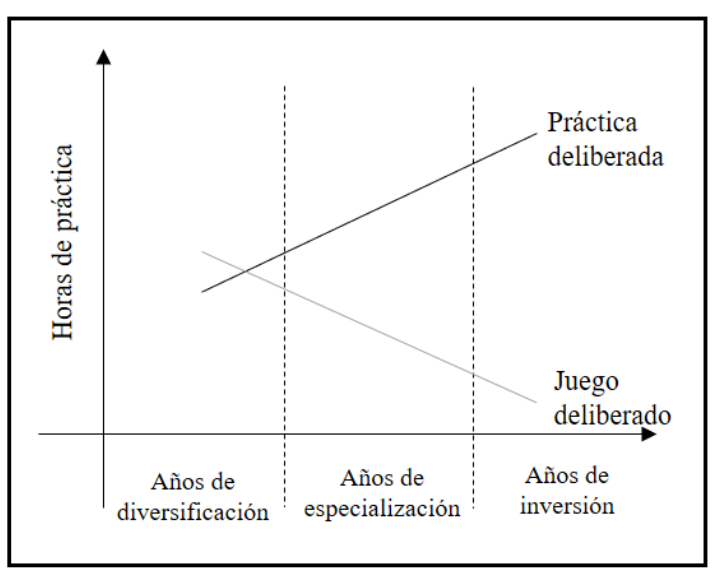

Representación gráfica de la formación deportiva observada en los atletas de élite del voleibol brasileño

Figura 2: Representación gráfica de la formación deportiva a partir del MDPD y la encontrada en jugadores de élite del voleibol brasileño.

El MDPD indica que, durante la diversificación, los jugadores deben involucrarse predominantemente con actividades de juego deliberado para que se forme una sólida base de control y diversificación motora, a partir de actividades que son intrínsecamente motivantes (Côté y col., 2009). En el presente estudio, los jugadores indicaron que participaron de actividades de juego deliberado en esta etapa, pero que no fueron significativamente predominantes. Resultados similares fueron encontrados con jugadores de fútbol (Diogo y Gonçalves, 2014), y de deportes colectivos (Forsman y col., 2016), en que la participación desde temprano con actividades estructuradas puede haber favorecido la familiarización con el proceso de desarrollo de la pericia deportiva. La investigación realizada con jugadores de selecciones nacionales de Alemania reveló que aquellos que obtuvieron éxito temprano en la carrera deportiva fueron los que iniciaron su especialización precoz, pero los jugadores que alcanzaron el nivel internacional en la edad sénior fueron los que se especializaron más tarde, 14 años (Gullich y Emrich, 2014). En este sentido, se ha identificado una correlación 
Collet, C.; Nascimento, J. V.; Folle, A.; Mendes, F. G., y Ibañez, S. J. (2018). Juego y práctica deliberada en la formación deportiva de jugadores de élite del voleibol. RICYDE. Revista internacional de ciencias del deporte. 54(14), 321-333. https://doi.org/10.5232/ricyde2018.05403

negativa estadísticamente significativa entre la participación, especialización, en las selecciones nacionales de base de baloncesto, y su posterior participación en la selección absoluta y equipos profesionales (Ibáñez, Sáenz-López, Feu, Giménez, y García, 2010).

Las actividades lúdicas, presentes en equilibrio con las actividades de entrenamiento en la primera etapa, disminuyeron gradualmente en la segunda y tercera etapas, en contrapartida, hubo un aumento en el tiempo de actividades de práctica deliberada que buscaban el perfeccionamiento deportivo. De acuerdo con las orientaciones del MDPD, en los años de especialización debe haber equilibrio entre la participación en actividades de juego y de práctica deliberada, así como posibilitar la elección por la especialización en su deporte favorito o la continuación en el deporte recreacional (Côté, 1999; Côté y col., 2007; Côté y Fraser-Thomas, 2007; Côté y col., 2009). Sin embargo, la predominancia de horas de actividades de práctica deliberada en esa etapa puede haber influido en el éxito precoz y en la convocatoria de los jugadores para las selecciones más jóvenes, que son la gran parte en el presente estudio. Estos resultados también se observaron en los estudios con jugadores finlandeses de fútbol, hockey sobre hielo y baloncesto (Forsman y col., 2016) y con jugadores alemanes de nivel nacional (Gullich y Emrich, 2014).

En los años de inversión, se observó gran número de actividades de práctica y pocas horas de juego deliberado, correspondiendo a las indicaciones del modelo y asimilando a los resultados de estudios con jugadores de hockey sobre hielo (Soberlak y Côté, 2003), voleibol (Coutinho y col., 2016) y deportes colectivos (Baker, Côté y Abernethy, 2003b).

Algunos estudios realizados con jugadores expertos revelaron que hay una intensificación de la participación en las actividades de práctica deliberada y con todo el contexto y situaciones que ayudan en el desarrollo de la pericia deportiva, como participar en competiciones, entrenamiento individualizado, y ver videos de juegos y entrenamientos (Baker y col., 2003a, Pazo, Sáenz-López y Fradua, 2012), entre otros factores determinantes para el alcance de la experiencia (Sáenz-López, Ibáñez, Giménez, Sierra y Sánchez, 2005). En este sentido, el entrenamiento estructurado, sumado a otros factores influyentes, contribuye a la formación de jugadores de élite, teniendo presente que se constituye de un proceso orientado hacia el desarrollo de habilidades para la pericia deportiva, que culminan en los momentos de competición.

En esta perspectiva, participar de diferentes tipos de actividades puede contribuir al desarrollo de la pericia deportiva. Sin embargo, hay que tener en cuenta las características del deporte, los contextos y las oportunidades de práctica, que también son importantes en este proceso. Existen diferencias específicas entre los países, dependiendo de los aspectos culturales y sociales, en lo que se refiere al desarrollo de programas deportivos (Forsman y col., 2016). En el caso de Brasil, el proceso de mercantilización del voleibol, iniciado en los años ochenta, permitió conseguir altas inversiones de patrocinadores e impulsó el desarrollo de la modalidad (Miranda, Pedroso, Barros-Filho, Silva y Rocha, 2016), principalmente en la región sudeste, donde se concentran los principales clubes formadores del país. La gran diversidad contextual en Brasil, en virtud de su alcance territorial y grandes desigualdades sociales, interfiere directamente en la práctica deportiva y en el desarrollo de jugadores, en que las oportunidades de alcance de la experiencia están condicionadas a la movilidad hacia grandes centros urbanos. A pesar de la importancia del tema, tales aspectos no fueron tenidos en cuenta en el estudio, debido a su alcance y el objetivo de enfocarse en el tipo de actividades (juego deliberado y práctica deliberada) realizadas por los jugadores de élite del voleibol. 
Collet, C.; Nascimento, J. V.; Folle, A.; Mendes, F. G., y Ibañez, S. J. (2018). Juego y práctica deliberada en la formación deportiva de jugadores de élite del voleibol. RICYDE. Revista internacional de ciencias del deporte. 54(14), 321-333. https://doi.org/10.5232/ricyde2018.05403

\section{Conclusiones}

La formación deportiva de los jugadores de élite del voleibol brasileño sigue un modelo específico, condicionado por las características territoriales, culturales y de política deportiva, que guarda ciertas semejanzas con el MDPD.

La formación se produjo a partir de la participación inicial con actividades de juego y práctica deliberada, con aumento gradual en la duración semanal de las actividades de práctica deliberada y disminución del juego deliberado. Este escenario corresponde a la indicación de la literatura internacional, que destaca las influencias positivas de las actividades de ocio en la etapa inicial para la posterior implicación con el ambiente de práctica sistematizada y la búsqueda por el rendimiento deportivo.

En contrapartida, el momento en que esa transición ocurrió en los jugadores brasileños se diferenció de las etapas iniciales sugeridas por la literatura. El equilibrio entre las actividades de práctica deliberada y juego deliberado ocurrió en la etapa inicial de formación deportiva de los jugadores de voleibol (diversificación), cuando las indicaciones son de empezar en la segunda etapa (especialización). El alcance territorial del país, con diferentes culturas, oportunidades y políticas de desarrollo deportivo, que afectan directamente la participación de los niños y jóvenes con el deporte.

A pesar del número limitado de jugadores participantes del estudio, fue posible identificar algunas características acerca de la participación con actividades de juego y práctica deliberada en cada etapa de la formación deportiva de élite del voleibol brasileño. En ese sentido, fue posible identificar algunos aspectos que podrán ayudar en la creación de un modelo de formación más adecuado este contexto.

\section{Agradecimientos}

Agradecimiento a la Coordinación de Perfeccionamiento de Personal de Nivel Superior / Brasil por la concesión de las becas CAPES DS y PDSE.

\section{Referencias}

Aiken, L. (1985). Three coeffients for analyzing the reability and validity of ratings. Educational and Psychological Measurement, 45(1), 131-143.

Ato, M.; López, J. J., y Benavente, A. (2013). Un sistema de clasificación de los diseños de investigación en psicología. Anales de Psicología, 29(3), 1038-1059. https://doi.org/10.6018/analesps.29.3.178511

Baker, J.; Côté, J., \& Abernethy, B. (2003a). Learning from the experts: Practice activities of expert decision-makers in sport. Research Quarterly for Exercise and Sport, 74(3), 342-347.

Baker, J.; Côté, J., \& Abernethy, B. (2003b). Sport-specific practice and the development of expert decision-making in team ball sports. Journal of Applied Sport Psychology, 15(1), 12-25.

https://doi.org/10.1080/10413200390180035

Baker, J., \& Young, B. (2014). 20 years later: deliberate practice and the development of expertise in sport. International Review of Sport and Exercise Psychology, 7(1), 135157.

https://doi.org/10.1080/1750984x.2014.896024

Berry, J.; Abernethy, B., \& Côté, J. (2008). The contribution of structured activity and deliberate play to the development of expert perceptual and decision-making skill. Journal of Sport \& Exercise Psychology, 30(6), 685-708. 
Collet, C.; Nascimento, J. V.; Folle, A.; Mendes, F. G., y Ibañez, S. J. (2018). Juego y práctica deliberada en la formación deportiva de jugadores de élite del voleibol. RICYDE. Revista internacional de ciencias del deporte. 54(14), 321-333. https://doi.org/10.5232/ricyde2018.05403

Box, G. E. P., \& Cox, D. R. (1964). An analysis of transformation. Journal of the Royal Statistical Society, 26(2), 211-252.

Cárdenas, M., y Arancibia, H. (2014). Potencia estadística y cálculo del tamaño del efecto en G*power: Complementos a las pruebas de significación estadística y su aplicación en psicología. Salud \& Sociedad, 5(2), 210-224.

Casado-Alda, A., y Ruiz-Pérez, L. M. (2017). Los corredores kenianos y españoles de larga distancia y su práctica deliberada. Revista de Psicologia del Deporte, 26(2), $55-$ 61.

Cohen, J. (1992). A Power Primer. Psychological Bulletin, 112(1), 155-159.

Côté, J. (1999). The influence of the family in the development of talent in Sport. The Sport Psychologist, 13(4), 395-417.

Côté, J., \& Abernethy, B. (2012). A Developmental Approach to Sport Expertise. In S. Murphy (Ed.), The Oxford handbook of sport and performance psychology (pp. 435447). New York: Oxford University Press.

Côté, J.; Baker, J., \& Abernethy, B. (2003). From play to practice: A developmental framework for the acquisition of expertise in team sport. In J. Starkes \& K. A. Ericsson (Eds.), Expert Performance in Sports: Advances in Research on Sport Expertise (pp. 89-113). Champaign, IL: Human Kinetics.

Côté, J.; Baker, J., \& Abernethy, B. (2007). Practice and play in the development of sport expertise. In R. Eklund \& G. Tenenbaum (Eds.), Handbook of Sport Psychology (3rd. ed., pp. 184-202). Hoboken/NJ: Wiley.

Côté, J., \& Erickson, K. (2015). Diversification and deliberate play during the sampling years. In J. Baker \& D. Farrow (Eds.), Routledge Handbook of Sport Expertise (pp. 305-316). London and New York: Routledge.

Côté, J., \& Fraser-Thomas, J. (2007). Youth involvement in sport. In P. Crocker (Ed.), Sport Psychology: a Canadian perspective (pp. 270-298). Toronto: Pearson.

Côté, J., \& Hay, J. (2002). Family influences on youth sport participation and performance. In J. Silva \& D. Stevens (Eds.), Psychological foundations of sport (pp. 503-519). Boston/MA: Allyn and Bacon.

Côté, J.; Lidor, R., \& Hackfort, D. (2009). To sample or to specialize? Seven postulates about youth sport activities and elite performance. International Journal of Sport and Exercise Psychology, 9(1), 7-17.

Côté, J.; Turnnidge, J., \& Vierimaa, M. (2016). A personal assets approach to youth sport. In K. Green \& A. Smith (Eds.), Routledge Handbook os Youth Sport (pp. 243255). London and New York: Routledge.

Côté, J., \& Vierimaa, M. (2014). The developmental model of sport participation: 15 years after its first conceptualization. Science \& Sports, 29(Supl.), 63-69. http://dx.doi.org/10.1016/j.scispo.2014.08.133

Coutinho, P.; Mesquita, I.; Davids, K.; Fonseca, A. M., \& Côté, J. (2016). How structured and unstructured sport activities aid the development of expertise in volleyball players. Psychology of Sport and Exercise, 25, 51-59. https://doi.org/10.1016/j.psychsport.2016.04.004

Diogo, F., \& Gonçalves, C. E. (2014). The path to expertise in youth sport: using a retrospective interview in three different competitive contexts. Perceptual \& Motor Skills: Exercise \& Sport, 118(2), 317-330.

Ericsson, K. A. (2003). Development of elite performance and deliberate practice. In J. L. Starkes \& K. A. Ericsson (Eds.), Expert Performance in Sports (pp. 50-83): Human Kinetics. 
Collet, C.; Nascimento, J. V.; Folle, A.; Mendes, F. G., y Ibañez, S. J. (2018). Juego y práctica deliberada en la formación deportiva de jugadores de élite del voleibol. RICYDE. Revista internacional de ciencias del deporte. 54(14), 321-333. https://doi.org/10.5232/ricyde2018.05403

Ericsson, K. A. (2006). The influence of experience and deliberate practice on the development of superior expert performance. In K. A. Ericsson, N. Charness, P. J. Feltovich, \& R. R. Hoffman (Eds.), The Cambridge handbook of expertise and expert performance (pp. 683-703). New York: Cambridge University Press.

Ericsson, K. A.; Krampe, R. T., \& Tesch-Romer, C. (1993). The Role of Deliberate Practice in the Acquisition of Expert Performance. Psychological Review, 100(3), 363406.

Field, A. (2009). Discoveryin Statistics Using SPSS. London: SAGE Publications.

Ford, P. R.; Ward, P.; Hodges, N. J., \& Williams, A. M. (2009). The role of deliberate practice and play in career progression in sport: the early engagement hypothesis. High Ability Studies, 20(1), 65-75.

https://doi.org/10.1080/13598130902860721

Forsman, H.; Blomqvist, M.; Davids, K., Konttinen, N., \& Liukkonen, J. (2016). The role of sport-specific play and practice during childhood in the development of adolescent Finnish team sport athletes. International Journal of Sports Science \& Coaching, 11(1), 69-77.

https://doi.org/10.1177/1747954115624816

Grissom, R. J., \& Kim, J. J. (2012). Effect sizes for research: univariate and multivariate applications. New York: Routledge - Taylor \& Francis Group.

Gullich, A. (2016). International medallists' and non-medallists' developmental sport activities - a matched-pairs analysis. Journal of Sports Science, 35(23), 2281-2288. https://doi.org/10.1080/02640414.2016.1265662

Gullich, A., \& Emrich, E. (2014). Considering long-term sustainability in the development of world class success. European Journal of Sport Science, 14(1), 383-397. https://doi.org/10.1080/17461391.2012.706320

Hendry, D. T.; Crocker, P. R., \& Hodges, N. J. (2014). Practice and play as determinants of self-determined motivation in youth soccer players. Journal of Sports Science, 32(11), 1091-1099.

https://doi.org/10.1080/02640414.2014.880792

Hornig, M.; Aust, F., \& Gullich, A. (2016). Practice and play in the development of German top-level professional football players. European Journal of Sport Science, 16(1), 96-105.

https://doi.org/10.1080/17461391.2014.982204

Ibáñez, S. J.; Sáenz-López, P.; Feu, S.; Giménez, J., \& García, J. (2010). Progression of Spanish National Team Basketball Players by Age and Sex. The Open Sports Sciences Journal (3), 118-128.

Memmert, D.; Baker, J., \& Bertsch, C. (2010). Play and practice in the development of sport-specific creativity in team ball sports. High Ability Studies, 21(1), 3-18. https://doi.org/10.1080/13598139.2010.488083

Mesquita, I. (1998). O ensino do voleibol: uma proposta metodológica. In A. Graça y J. Oliveira (Eds.), $O$ ensino dos jogos desportivos coletivos (pp. 153-199). Porto: FCDEF-UP.

Milistetd, M.; Mesquita, I.; Nascimento, J. V., y Sousa Sobrinho, A. E. P. (2010). Concepções de treinadores "experts" brasileiros sobre o processo de formação desportiva do jogador de voleibol. Revista Brasileira de Educação Física e Esporte, 24(1), 79-93. 
Collet, C.; Nascimento, J. V.; Folle, A.; Mendes, F. G., y Ibañez, S. J. (2018). Juego y práctica deliberada en la formación deportiva de jugadores de élite del voleibol. RICYDE. Revista internacional de ciencias del deporte. 54(14), 321-333. https://doi.org/10.5232/ricyde2018.05403

Miranda, Y. H. B.; Pedroso, C. A. M. Q.; Barros-Filho, M. A.; Silva, V. H. R., y Rocha, V. L. S. (2016). A importância da gestão esportiva no desenvolvimento do voleibol brasileiro: estratégias da Confederação Brasileira de Voleibol. Revista de Gestão e Negócios do Esporte, 1(1), 13-23.

Pazo, C. I.; Sáenz-López, P., y Fradua, L. (2012). Influencia del contexto deportivo en la formación de los futbolistas de la selección española de fútbol. Revista de Psicologia del Deporte, 21(2), 291-299.

Santos, A. J. F. O. (2011). O Percurso para a Excelência: Estudo de caso com a atleta de basquetebol "Ticha" Penicheiro. (Mestre em Treinamento de Alto Rendimento Esportivo Dissertação de mestrado), Universidade do Porto, Porto, Portugal.

Sáenz-López, P.; Ibáñez, S. J.; Giménez, F. J.; Sierra, A., \& Sánchez, M. (2005). Multifactor characteristics in the process of development of the male expert basketball placer in Spain. Internacional Journal of Sport Psychology, 36(2), 151-171.

Soberlak, P., \& Côté, J. (2003). The Developmental Activities of Elite Ice Hockey Players. Journal of Applied Sport Psychology, 15(1), 41-49.

http://dx.doi.org/10.1080/10413200305401

Vink, K.; Raudsepp, L., \& Kais, K. (2015). Intrinsic motivation and individual deliberate practice are reciprocally related: Evidence from a longitudinal study of adolescent team sport athletes. Psychology of Sport and Exercise, 16(3), 1-6.

http://dx.doi.org/10.1016/j.psychsport.2014.08.012

Ward, P.; Hodges, N. J.; Starkes, J. L., \& Williams, M. A. (2007). The road to excellence: deliberate practice and the development of expertise. High Ability Studies, 18(2), 119-153.

https://doi.org/10.1080/13598130701709715 